\title{
COMMENTARY
}

\section{Another brick in the wall of needs for invasive ventilation?}

\author{
Michael Quintel ${ }^{*}$ and Onnen Moerer \\ See related review by Mirabella et al., http://ccforum.com/content/18/1/R22
}

\begin{abstract}
Ventilator-induced lung injury and ventilator-induced diaphragmatic dysfunction are major complications in mechanically ventilated patients with acute respiratory failure. Invasive ventilation adds a further burden by increasing the risk of infections. An approach that protects both lung and diaphragm is pivotal. Mirabella and colleagues compared conventional controlled ventilation with a mode that combines several potentially lung-protective properties - non-invasively applied neurally adjusted ventilatory assist - in an animal experiment. This approach seemed to be as effective but potentially more lung-protective. Although the experimental setup and results cannot be translated directly to the clinical setting, they should motivate us to further study this innovative approach.
\end{abstract}

\section{Introduction}

In the previous issue of Critical Care, Mirabella and colleagues [1] studied the lung-protective capabilities of noninvasively applied neurally adjusted ventilatory assist (NAVA) in acute respiratory failure (ARF) in an animal study. In ARF, mechanical ventilation is applied to maintain adequate gas exchange, to stabilize the alveolar space, and to unload the respiratory muscles. The price to pay is, first and foremost, ventilator-induced lung injury (VILI). 'Protective' strategies using low tidal volumes and limited airway pressures in combination with positive endexpiratory pressure (PEEP) have been able to show a gradual reduction of VILI, thus influencing outcome [2]. In addition to VILI, controlled mechanical ventilation causes atrophic loss of muscle strength because of inactivation

\footnotetext{
* Correspondence: mquintel@med.uni-goettingen.de

Department of Anaesthesiology, Emergency and Intensive Care Medicine,

University Medicine, Georg-August-University Göttingen, Robert Koch Strasse 40, 37075 Göttingen, Germany
}

and ventilator-induced diaphragmatic dysfunction (VIDD) $[3,4]$. Both VILI and VIDD have major implications for morbidity and mortality.

NAVA provides airway pressures proportional to the electrical activity of the diaphragm during inspiration, ensures efficient patient-ventilator synchrony, and maintains the degree of biovariability that corresponds to the actual status of the patient [5-9]. These properties not only might be lung-protective but also might enable a more physiologic distribution of ventilation within the lungs, leading to improved matching of ventilation and perfusion.

The (at least) initial use of non-invasive ventilation (NIV) in hypercapnic ARF with respiratory muscle weakness is a well-accepted medical standard; in contrast, its use in hypoxemic failure (adult respiratory distress syndrome, or ARDS) is limited because even a very short loss of end-expiratory pressure might lead to significant derecruitment and severe hypoxemia [10]. Combining the effect of these two different approaches (NIVNAVA), the study by Mirabella and colleagues provides new conceptual insights into lung-protective ventilation [1].

\section{Hypothesis, results and open questions}

Based on the hypothesis that NIV-NAVA (without external PEEP) might be equally protective, the authors compared it with conventional invasive mechanical ventilation (tidal volume of $6 \mathrm{~mL} / \mathrm{kg}$ and PEEP of $5 \mathrm{~cm}$ $\mathrm{H}_{2} \mathrm{O}$ ) with neuromuscular blockade in an animal experiment with acid-induced ARF (arterial partial pressure of oxygen/fraction of inspired oxygen ratio of less than 200) [1]. At 6 hours, both groups recovered to oxygenation values found before injury. Only the NIV-NAVA group demonstrated an improvement in the dynamic compliance; consequently, lower peak and plateau pressures were measured and lung injury was less pronounced in NIV-NAVA as proven by inflammatory reaction and lung histology. 
The authors ascribe these effects to the maintenance of spontaneous breathing, upper airway liberation, and variable breathing pattern. The approach of this study is innovative and, to some extent, challenges the iron law that, in ARDS states comparable to those experimentally induced in this study, intubation and controlled mechanical ventilation are the only appropriate options.

The study, though presenting challenging results, has obvious limitations, foremost relating the findings to one well defined, specific intervention. This is true for two reasons. First, the study compares two different modes of mechanical ventilation: volume-controlled mechanical ventilation in paralyzed, deeply sedated subjects and assisted mechanical ventilation applied with NAVA. Second, it compares two different interfaces by which mechanical ventilation is applied: invasive mechanical ventilation via an endotracheal tube and NIV applied via a nasal prong.

A group of non-invasive pressure support ventilated animals would have allowed us to better understand and interpret the results, but (as stated by the authors) this was technically not achievable.

Before open NIV-NAVA can be considered in the clinical setting, a number of questions presented by the complexity of the topic have to be answered:

1. Do the positive effects prevail over a longer period of time? In a setting where positive effects have to persist and side effects might need time to develop their detrimental effects, an observation period of 6 hours is too short.

2. Is persistent tonic activity less injurious than the application of PEEP? PEEP has a direct effect on respiratory muscle load, recruitment, and pulmonary gas exchange; it reduces alveolar cycling and influences the respiratory drive [11]. Tonic activity of the diaphragm describes a persistently increased activity during the expiratory phase $[12,13]$. The data presented suggest that, in a setting without external PEEP, increased tonic diaphragmatic activity enables recruitment and enlarges end-expiratory lung volume. The observation that increased tonic diaphragmatic activity induced effects comparable to those of controlled mechanical ventilation with a PEEP of $5 \mathrm{~cm} \mathrm{H}_{2} \mathrm{O}$ underlines the importance of non-prima vista effects of NAVA that might open new treatment options for respiratory failure. If ARF resolves within a short period of time, preservation of tonic activity might be an effective measure. The long-term effect remains to be elucidated. A prolonged phase of induced tonic activity might fatigue the diaphragm and induce secondary respiratory failure [13]. The increased tonic activity toward the end of the experiment, therefore, should be interpreted with caution. It remains unclear whether open NIVNAVA outperforms a setting that includes externally applied PEEP. Consequently, Mirabella and colleagues do not suggest the use of zero PEEP in the clinical setting.

3. Are the findings transferable to a more stable severe ARDS and increased respiratory drive? The preservation of spontaneous breathing has marked beneficial effects [14]. However, by increasing the forces applied to the lung, an unsuppressed, severely increased respiratory drive might be harmful [15]. In states of severe hypoxemia, the question of whether to put the patient at rest or to admit his or her own respiratory drive remains unanswered.

Twenty percent regurgitation in the NIV group would be unacceptable in clinical routine. This finding, however, should be interpreted with caution since the sedation level required to enable the animal experiment might account for this side effect.

\section{Conclusions}

This experimental study by Mirabella and colleagues offers interesting insights into the potentials of NIVNAVA [1]. It is encouraging that NIV-NAVA has effectively maintained a state in which pulmonary gas exchange was seemingly more protective than conventional invasive ventilation. Thus, in a reversible injury with fast recovery and low tendency for derecruitment, open NIV-NAVA seems beneficial since it offers the opportunity to avoid controlled mechanical ventilation and intubation. Being applied with uncontrollable leakage underlines the properties and potentials of NAVA and should encourage further studies with NIV-NAVA to define its indications in the clinical setting.

However, we would like to state that this study challenges accepted limits or standard of care in several ways. In light of the current standard of routine care in patients who resemble the chosen experimental setup (moderate ARDS), the findings should be weighed carefully before being translated to clinical practice. As noted by the authors themselves, NIV should be restrictively used in patients with hypoxemic respiratory failure, and its application without PEEP cannot be advised at this stage.

\footnotetext{
Abbreviations

ARDS: Adult respiratory distress syndrome; ARF: Acute respiratory failure; NAVA: Neurally adjusted ventilatory assist; NIV: Non-invasive ventilation; PEEP: Positive end-expiratory pressure; VIDD: Ventilator-induced diaphragmatic dysfunction; VILI: Ventilator-induced lung injury. 
Critical Care (Rastatt, Germany), and is remunerated for these consultations. In 2006, OM participated in a research project at Uppsala University, financed by research grants from Göttingen and Uppsala University and supported by Maquet Critical Care.

Published: 17 Mar 2014

\section{References}

1. Mirabella L, Grasselli G, Haitsma JH, Zhang H, Slutsky AS, Sinderby C, Beck J: Lung injury during non-invasive synchronized assist versus volume control in rabbits. Crit Care 2014, 18:R22.

2. : Ventilation with lower tidal volumes as compared with traditional tidal volumes for acute lung injury and the acute respiratory distress syndrome. The Acute Respiratory Distress Syndrome Network. N Engl J Med 2000, 342:1301-1308.

3. Jaber S, Jung B, Matecki S, Petrof BJ: Clinical review: ventilator-induced diaphragmatic dysfunction - human studies confirm animal model findings! Crit Care 2011, 15:206.

4. Levine S, Nguyen T, Taylor N, Friscia ME, Budak MT, Rothenberg P, Zhu J, Sachdeva R, Sonnad S, Kaiser LR, Rubinstein NA, Powers SK, Shrager JB: Rapid disuse atrophy of diaphragm fibers in mechanically ventilated humans. N Engl J Med 2008, 358:1327-1335.

5. Sinderby C, Navalesi P, Beck J, Skrobik Y, Comtois N, Friberg S, Gottfried SB, Lindström $L:$ Neural control of mechanical ventilation in respiratory failure. Nat Med 1999, 5:1433-1436.

6. Moerer O: Effort-adapted modes of assisted breathing. Curr Opin Crit Care 2012, 18:61-69.

7. Spahija J, de Marchie M, Albert M, Bellemare P, Delisle S, Beck J, Sinderby C: Patient-ventilator interaction during pressure support ventilation and neurally adjusted ventilatory assist. Crit Care Med 2010, 38:518-526.

8. Piquilloud L, Vignaux L, Bialais E, Roeseler J, Sottiaux T, Laterre PF, Jolliet P, Tassaux D: Neurally adjusted ventilatory assist improves patientventilator interaction. Intensive Care Med 2011, 37:263-271.

9. Colombo D, Cammarota G, Bergamaschi V, De Lucia M, Corte FD, Navales P: Physiologic response to varying levels of pressure support and neurally adjusted ventilatory assist in patients with acute respiratory failure. Intensive Care Med 2010-2018, 2008:34.

10. Neumann P, Berglund JE, Mondejar EF, Magnusson A, Hedensteirna G: Effect of different pressure levels on the dynamics of lung collapse and recruitment in oleic-acid-induced lung injury. Am J Resp Crit Care Med 1998, 158:1636-1643.

11. Passath C, Takala J, Tuchscherer D, Jakob SM, Sinderby C, Brander L: Physiologic response to changing positive end-expiratory pressure during neurally adjusted ventilatory assist in sedated, critically ill adults. Chest 2010, 138:578-587.

12. Ducharme-Crevier L, Du Pont-Thibodeau G, Emeriaud G: Interest of monitoring diaphragmatic electrical activity in the pediatric intensive care unit. Crit Care Res Pract 2013, 2013:384210.

13. Emeriaud G, Beck J, Tucci M, Lacroix J, Sinderby C: Diaphragm electrical activity during expiration in mechanically ventilated infants. Pediatr Res 2006, 59:705-710.

14. Putensen C, Mutz NJ, Putensen-Himmer G, Zinserling J: Spontaneous breathing during ventilatory support improves ventilation-perfusion distributions in patients with acute respiratory distress syndrome. Am J Respir Crit Care Med 1999, 159:1241-1248.

15. Yoshida T, Uchiyama A, Matsuura N, Mashimo T, Fujino Y: Spontaneous breathing during lung-protective ventilation in an experimental acute lung injury model: high transpulmonary pressure associated with strong spontaneous breathing effort may worsen lung injury. Crit Care Med 2012, 5:1578-1585.

$10.1186 / \mathrm{cc} 13769$

Cite this article as: Quintel and Moerer: Another brick in the wall of needs for invasive ventilation? Critical Care 2014, 18:122 